\title{
Role of Fine Needle Aspiration in The Diagnosis of Metaplastic Carcinoma of Breast
}

\author{
Sree Rekha Jinkala*, Siddaraju Neelaiah and Bhawana A Badhe
}

Department of Pathology, Institute Block, Jawaharlal Institute of Postgraduate Medical Education and Research (JIPMER), Gorimedu, Puducherry, India

\begin{abstract}
Metaplastic carcinomas of breast are rare primary malignant tumors of breast with high rate of recurrences and metastasis. It poses diagnostic difficulties in histopathology as well as in cytopathology because of its varied morphological features. We present a case of a 54 year old woman diagnosed as metaplastic carcinoma of breast by fine needle aspiration and subsequently by histopathology. Our case shows predominantly mesenchymal components like spindle shaped cells, chondroid matrix material and numerous osteoclastic giant cells along with a scanty malignant epithelial component, which was better appreciated on the FNA material, by which a definitive diagnosis of metaplastic carcinoma of breast was possible on FNA. The epithelial component was underrepresented in the subsequent trucut biopsy, thus emphasizing the adjuvant role of FNA in diagnosing these tumors. In this report we discuss the challenges involved in diagnosing metaplastic carcinoma of breast on FNA.
\end{abstract}

Keywords: Metaplastic Carcinoma; Breast, Fine Needle Aspiration, Epithelial, Mesenchymal, Spindle Cells.

\section{Introduction}

Metaplastic carcinomas of breast are rare primary aggressive neoplasms of breast which show both epithelial and mesenchymal components. They account for $<1 \%$ malignant breast tumors. ${ }^{[1]}$ Histopathology shows varied morphological features, sometimes causing diagnostic difficulties. Diagnosis of metaplastic carcinoma of breast by fine needle aspiration (FNA) cytology has its own limitations; but many a times it is due to lack of proper representation of diagnostic material or sparse yield of cytological material. We report a case of metaplastic carcinoma of breast which showed mesenchymal as well as epithelial components only on cytologic material.

\section{Case Report}

A 54 year old nulliparous postmenopausal woman presented with a lump in the right breast of one month duration. On examination, a $5 \times 5 \mathrm{~cm}$ firm mobile lump was present in upper outer quadrant of right breast. The skin over the lump showed erythema, however the nipple areola complex was unremarkable. A 1x1 cm lymphnode was present in the right axillary region. FNA was done from breast lump with a $23 \mathrm{G}$ needle attached to a $10 \mathrm{ml}$ syringe and yielded blood mixed particulate material. Smears were stained with May Grundwald-Geimsa (MGG) and Papaniculoau (PAP) stains. Subsequently a trucut biopsy from the breast lump has been done. The biopsy was formalin fixed and paraffin embedded; five micron thick sections stained with Haematoxylin and Eosin were studied.
Cytological Findings: Smears showed tissue fragments composed of spindle shaped cells, along with few loose clusters and single lying spindle cells with the periphery of the fragments showed osteoclastic gaint cells (Figure 1a). These spindle cells showed increased nucleo-cytoplasmic ratio, mild to moderate nuclear pleomorphism, vesicular nucleus, prominent nucleoli and bipolar cytoplasmic processes (Figure 1b). In addition, areas of chondroid matrix with chondrocytes were identifiable at many foci. The chondriod areas were pale eosinophilic amorphous looking on PAP smears (Figure 2a) and had purplish metachromatic nature on MGG (Figure 2b) . Atypical mitotic activity was frequent. The ductal epithelial component was seen as occasional small clusters with focal cribriform pattern ( Figure 1c) and single polygonal cells. At places the epithelial component was intimately seen with osteoclastic giant cells. The epithelial cells were polygonal with increased nucleocytoplasmic ratio,moderate to scant cytoplasm and coarse chromatin; occassional foci showed gland formation (Figure 1d). All these features led to a diagnosis of metaplastic carcinoma of breast on FNA material.

Histopathological Findings: Trucut biopsy showed multiple cores of tissue predominantly showing mesenchymal component comprising of spindle shaped cells arranged in fascicles with mild to moderate nuclear pleomorphism (Figure 2c). Cartilaginous areas (Figure 2c) as well as osseous metaplasia and osteoclastic giant cells were seen. In addition there were areas showing 
malignant osteoid lined by the tumor cells (Figure $2 \mathrm{~d}$ ). The epithelial component was underrepresented in the biopsy. As malignant epithelial component was seen on FNA material, a final histopathological diagnosis of metaplastic carcinoma of breast with a component of heterologous osteosarcoma has been made, after correlating with the cytological findings. Estrogen, progesterone receptor and Her2 neu were negative in the tumor.

\section{Discussion}

Metaplastic carcinoma of the breast (MBC) is a rare, heterogeneous disease consisting of tumors with admixed epithelial and non-epithelial elements. The non-epithelial elements can consist of spindled stromal cells, bizarre stromal giant cells, cartilage, bone, and rarely skeletal muscle, in varying proportions. ${ }^{[2,3]}$

Pathological classification of these tumors is challenging because of varied histomorphological features. The single largest series on MBC by Wargotz et al. suggested five variants of metaplastic carcinoma - matrix-producing carcinoma, squamous cell carcinoma, spindle cell carcinoma, carcinosarcoma, and metaplastic carcinoma with osteoclastic giant cells. ${ }^{[4]}$ Node negative MBC shows increased rate of recurrence compared to infiltrating ductal carcinoma, NOS. ${ }^{[5]}$ Some tumors classified as MBC behave like pure sarcomas with absence of regional lymph node involvement, aggressive vascular pattern of metastasis, and predilection for pulmonary involvement. ${ }^{[6]}$

MBC shows varied morphologic features not only on histopathology, but on cytology also. The cytologic smears may show areas of 1) atypical squamoid cells with necrosis in the background along with ductal epithelial cell component, 2) predominantly spindle shaped cells exhibiting various degrees of pleomorphism 3) matrix material like osseous or chondroid areas and osteoclastic like giant cells.

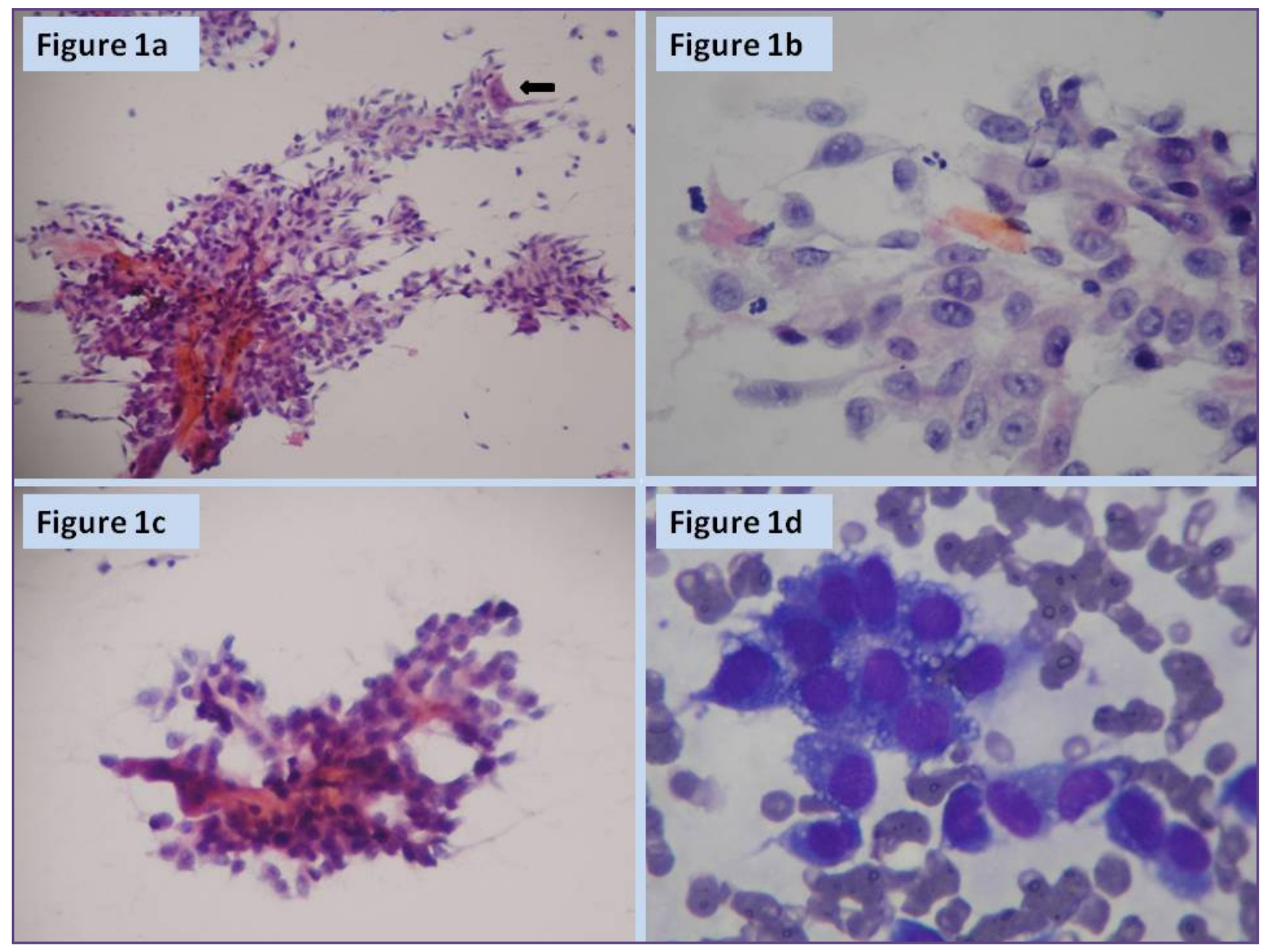

Fig. 1: Tissue fragments showing spindle cells with attached osteoclastic giant cells,1a (arrow) (PAP X100); and frequent mitosis, 1b (PAP X 400); Epithelial component with focal cribriform pattern, 1c (PAP X 100) and gland formation, 1d (MGG X 400). 


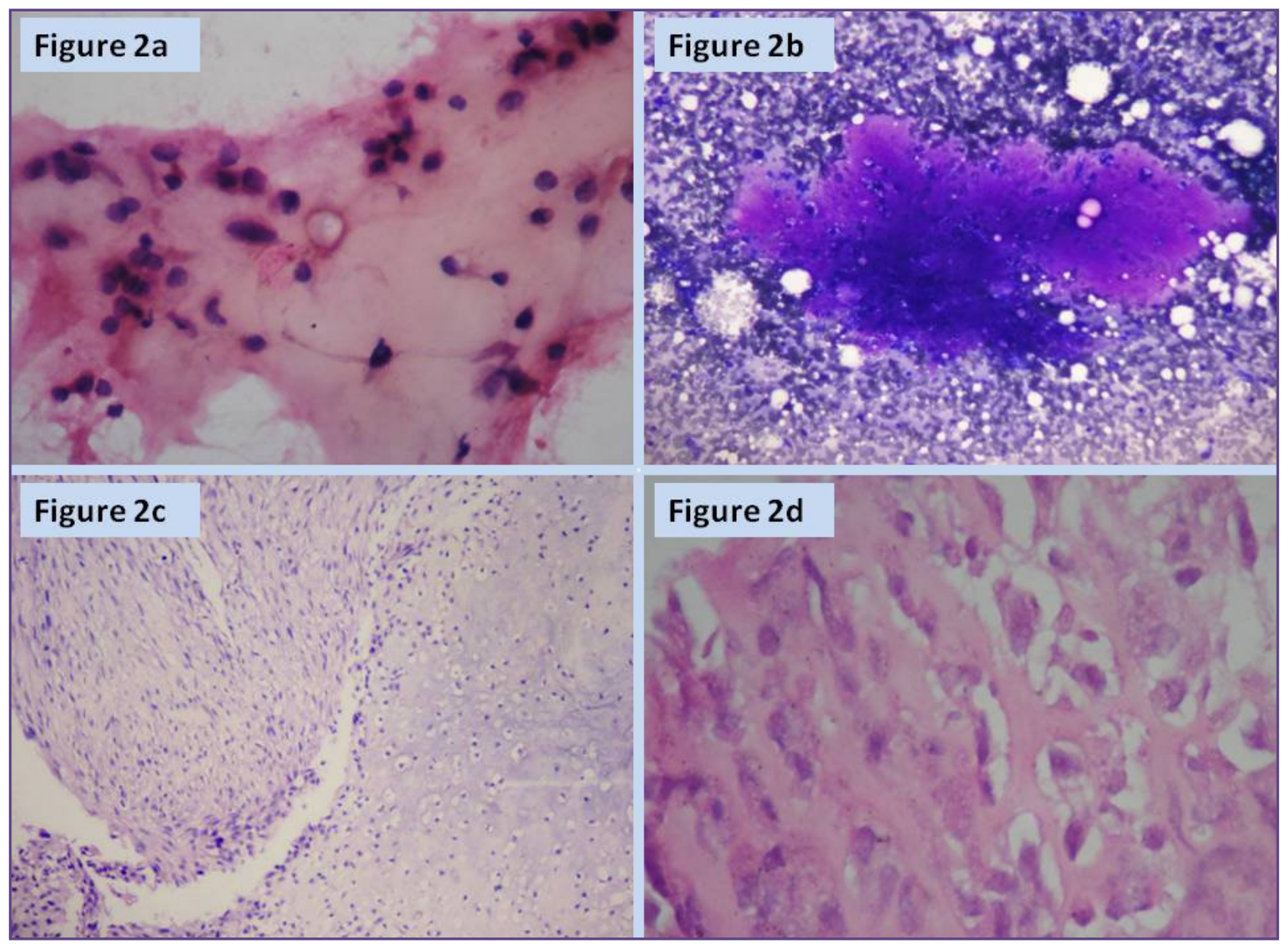

Fig. 2: Chondriod areas showing pale eosinophilic amorphous appearance on PAP, 2a (400X) and purplish metachromatic nature on MGG, 2b (100X); Trucut biopsy showing spindle shaped cell in fascicles, with chondroid areas (2c) and osteoid produced by the tumor cells (2d) (H\&E X 400).

Atypical squamoid cells are seen on breast FNA in metaplastic carcinoma with both adeno and squamous components, any entity with metaplastic changes such as phyllodes tumor or, in ductal carcinoma with in situcomedo carcinoma. ${ }^{[7]}$ In rare instances, sarcomas with radiation induced atypia can also show atypical squamoid cells. Primary and metastatic squamous cell carcinoma arising in the breast are very uncommon, but are well documented.

If the cytological material comprises of predominantly spindle cell component, the differentials of fibromatosis, nodular fasciitis, inflammatory myofibroblastic tumor (IMFT), phyllodes tumor with stromal overgrowth, metaplastic carcinoma, primary sarcoma of the breast and metastases should be considered. ${ }^{[9]}$

If the cytological material, in addition to the spindle component shows matrix material like osseous or chondroid material and osteoclastic giant cells, along with a malignant epithelial component, a diagnosis of $\mathrm{MBC}$ is almost certain as in our case. It needs to be correlated with histopathology which is the gold standard for diagnosis of MBC. If the malignant epithelial component is absent, primary sarcoma of the breast cannot be ruled out on cytologic material. Our case also emphasizes the need to look for epithelial component in these cases which was not evident on the H\&E section. Thus cytology has an important adjuvant role to biopsy in such cases as cytologic material can be obtained from different sites.

In many cases 'sampling' impacts the fine needle aspiration diagnosis. Multiple needle passes and sampling of all areas of the lesion are ideal to ensure an accurate cytologic diagnosis, but in reality compromises often times must be made in response to patient comfort. ${ }^{[7]}$ Inadequate sampling leads to under-representation of the varied components of the tumor which precludes the definitive diagnosis of $\mathrm{MBC}$. 


\section{Conclusion}

Definitive diagnosis of metaplastic carcinoma breast is possible by FNA and it also acts as an important adjuvant to histopathology in diagnosing metaplastic carcinomas.

\section{References}

1. Tavassoli FA.Classification of metaplastic carcinomas of the breast. Pathol Annual 1992; 27 : 89-119

2. Oberman HA. Metaplastic carcinoma of the breast. Am J Surg Path 1987; 11: 351-8

3. Foschini MP, Dina RE, Eusebi V. Sarcomatoid neoplasms of the breast: proposed definitions for biphasic and monophasic sarcomatoid mammary carcinomas. Seminars in Diag Pathol 1993;10: 128-36

4. Wargotz ES, Deos PH, Norris HJ. Metaplastic carcinomas of the breast. II. Spindle cell carcinoma. Hum Pathol 1989; 20:732-40
5. Barnes PJ, Boutilier R, Chaisson D, Rayson D. Metaplastic breast carcinoma: Clinico-pathological characteristics and Her2/Neu expression. Breast cancer research and treatment 2005; 91:173-8

6. Rayson D, Adjei AA, Suman VJ, Wold LE, Ingle JN. Metaplastic breast cancer: Prognosis and response to systemic therapy. Annal Oncol 1999;10: 413-9.

7. Lale S, Kure K, Lingamfelter D. Challenges to diagnose metaplastic carcinoma of the breast through cytologic methods: an eight-case series. Diagnostic Pathology 2011; 6:7.

8. Carter MR, Hornick JL, Lester S, Fletcher CD: Spindle cell (sarcomatoid) carcinoma of the breast: a clinicopathologic and immunohistochemical analysis of 29 cases. Am J Surg Pathol 2006; 30:300-9.

9. Akerman M, Domnski H. Soft tissues. Orell SR, Sterrett GF. Orell and Sterretts Fine needle aspiration cytology. $5^{\text {th }}$ edition: Churchill Livingstone, Elesiver Ltd. Pg 387-411.

*Corresponding author:

Dr Sree Rekha Jinkala, Assistant professor, Department of Pathology, Jawaharlal Institute of Postgraduate Medical Education and Research (JIPMER),

Gorimedu, Puducherry, India - 605006

Email: sree.path177@gmail.com

Financial or other Competing Interests: None. 